\title{
CORRELATION IMAGING IN INVERSE SCATTERING IS TOMOGRAPHY ON PROBABILITY DISTRIBUTIONS
}

\author{
PEDRO CARO ${ }^{1}$, TAPIO HELIN ${ }^{2}$, ANTTI KUJANPÄ̈${ }^{2}$, AND MATTI LASSAS $^{2}$
}

\begin{abstract}
Scattering from a non-smooth random field on the time domain is studied for plane waves that propagate simultaneously through the potential in variable angles. We first derive sufficient conditions for stochastic moments of the field to be recovered from empirical correlations between amplitude measurements of the leading singularities, detected in the exterior of a region where the potential is almost surely supported. The result is then applied to show that if two sufficiently regular random fields yield the same correlations, they have identical laws as function-valued random variables.
\end{abstract}

\section{INTRODUCTION}

Randomness is often an inherent part of any computational model for an applied inverse problem. For instance, it can reflect the chaotic evolution of the system or the perspective that the unknown object of interest is rough and vastly complex. Ultimately, the observational noise is most often probabilistic in nature. If the statistics of the system can be described to a good approximation, it can be desirable to transform the problem paradigm by considering correlations or other statistical moments of the data distribution and how that information relates to the relevant system parameters. This approach is often called correlation based imaging in literature and it has been recently studied for a variety of inverse problems (see e.g. applications in seismic imaging [1]).

In this paper we consider scattering of waves from a time-independent random potential $V$ supported on a fixed compact set in $\mathbb{R}^{n}$. We study an inverse problem of recovering the law of $V$ given certain empirical correlations in the exterior of the potential. The wave propagation is governed by

$$
\begin{aligned}
(\square-V(x)) u(x, t, \boldsymbol{\theta}) & =0, \\
u(x, t, \boldsymbol{\theta}) & =\sum_{j=1}^{k} \delta\left(t-x \cdot \theta_{j}\right)+u_{\mathrm{sc}}(x, t, \boldsymbol{\theta}), \\
u_{\mathrm{sc}}(x, t, \boldsymbol{\theta}) & =0, \quad \text { for } t \ll 0,
\end{aligned}
$$

where $(x, t) \in \mathbb{R}^{n+1}, \boldsymbol{\theta}=\tilde{\boldsymbol{\theta}}^{k}:=\left(\theta_{1}, \ldots, \theta_{k}\right), \square:=\partial_{t}^{2}-\Delta$ is the wave operator, and the potential

$$
V: \mathbb{R}^{n} \times \Omega \rightarrow \mathbb{R}, V(x)=V(x, \omega)
$$

\footnotetext{
${ }^{1}$ BCAM - Basque Center for Applied Mathematics, Alameda de Mazarredo 1448009 Bilbao, Bizkaia (BAsque-Country, Spain)

2 Department of Mathematics and Statistics, University of Helsinki, Gustaf Hällströmin KATU 2B, FI-00014 Helsinki, Finland

E-mail addresses: pcaro@bcamath.org, tapio.helin@helsinki.fi, antti.kujanpaa@helsinki.fi, matti.lassas@helsinki.fi.
} 
is a random generalized function, that is, a measurable map from the probability space $\Omega$ into a linear subspace of generalized functions which, in this paper, will be contained in the Sobolev space $H^{2}\left(\mathbb{R}^{n}\right):=W^{2,2}\left(\mathbb{R}^{n}\right)$ endowed with the Borel $\sigma$-algebra. We shall omit the parameter $\omega \in \Omega$ from notation and write $V(x)$ instead of $V(x, \omega)$.

Given a family of directions $\theta_{1}, \ldots, \theta_{k} \in \mathbb{S}^{n-1}$ which are not necessarily distinct from each other, let us denote the trajectory of the plane wave $\delta\left(t-\theta_{j} \cdot x\right), j \in\{1, \ldots, k\}$ by

$$
\Sigma_{j}:=\left\{(x, t) \in \mathbb{R}^{n} \times \mathbb{R}: x \cdot \theta_{j}=t\right\} .
$$

In the following $\Sigma_{j}(t)$ stands for the $(n-1)$-dimensional hyperplane in $\Sigma_{j}$ given a fixed $t \in \mathbb{R}$. It is well-known that after suitable time $T>0$ the potential $V$ produces a discontinuity in the scattered field across the hyperplane $\Sigma_{j}(t)$ for $t>T$. More importantly, the discontinuities carry information regarding the integral values of $V$ over the bicharacteristic lines. By setting measurement devices outside the region where the potential is almost surely supported one captures "the shadows" of the potential in different angles from the outgoing wave fronts as they collide into the detectors. After repeating the observation one can consider empirical correlations between patterns captured by separate measurement devices.

Motivated by this line of thought, we study correlations in the exterior of the potential. We assume that the observations approximate the following generalised function to a good approximation. We will refer to it as the empirical correlations:

$$
(\mathbf{x}, \boldsymbol{\theta}) \mapsto D^{k}(\mathbf{x}, \boldsymbol{\theta})=D_{V}^{k}(\mathbf{x}, \boldsymbol{\theta}) \quad \text { for all }(\mathbf{x}, \boldsymbol{\theta}) \in\left(\prod_{j=1}^{k}\left(\mathbb{R}^{n} \times \mathbb{S}^{n-1}\right)\right) \backslash \Xi,
$$

where $\mathbf{x}=\left(x_{1}, \ldots, x_{k}\right), \boldsymbol{\theta}=\left(\theta_{1}, \ldots, \theta_{k}\right)$,

$$
\Xi:=\left\{(\mathbf{x}, \boldsymbol{\theta}) \in \prod_{j=1}^{k}\left(\mathbb{R}^{n} \times \mathbb{S}^{n-1}\right): \theta_{j}=\theta_{l}, \text { for some } j, l \in\{1, \ldots, k\} \text { such that } j \neq l\right\}
$$

and

$$
D^{k}(\mathbf{x}, \boldsymbol{\theta}):=\mathbb{E}\left(\lim _{s \rightarrow \infty} \prod_{j=1}^{k}\left[u_{s c}\right]_{\Sigma_{j}}\left(x_{j}+s \theta_{j}, \boldsymbol{\theta}\right)\right) .
$$

It will be shown that the empirical correlations $D^{k}(\mathbf{x}, \boldsymbol{\theta})$ can be naturally extended to a generalised function on the whole space $\prod_{j=1}^{k}\left(\mathbb{R}^{n} \times \mathbb{S}^{n}\right)$. The notation $\left[u_{s c}\right]_{\Sigma_{j}}(x, \boldsymbol{\theta})$ stands for the jump across $\Sigma_{j}$,

$$
\left[u_{s c}\right]_{\Sigma_{j}}(x, \boldsymbol{\theta})=\left(\operatorname{Tr}_{\Sigma_{j}}^{+}-\operatorname{Tr}_{\Sigma_{j}}^{-}\right) u_{s c}\left(x, x \cdot \theta_{j}, \boldsymbol{\theta}\right),
$$

in which the traces $\operatorname{Tr}_{\Sigma_{j}}^{ \pm}$stand for restrictions to the boundary $\Sigma_{j}$ from the upper and lower half-spaces

$$
\left\{(x, t) \in \mathbb{R}^{n+1}: 0 \leq \pm\left(t-x \cdot \theta_{j}\right), x \in \mathbb{R}^{n} \backslash \overline{B(0, R)}\right\}
$$

and the radius $R$ is chosen large enough so that the support of the potential is almost surely contained in the ball $B(0, R)$. We give a precise definition of the the restrictions in the beginning of the next section.

The model (1) can also be interpreted in the frequency domain under the high frequency regime. Taking Fourier transform with respect to time converts the model into a timeharmonic system,

$$
\left(\Delta+\lambda^{2}+V(x)\right) \widehat{u}(x, \lambda, \boldsymbol{\theta})=0,
$$


associated with energy $\lambda^{2}$, an electric potential $V$, the incident wave $\widehat{u}_{I}(x, \lambda, \boldsymbol{\theta})=\sum_{j=1}^{k} e^{-i \lambda x \cdot \theta_{j}}$ and the Sommerfeld radiation condition. In quantum mechanics such a model typically arises from the time-dependent Schrödinger equation,

$$
(V(x)-\Delta) \Psi(x, s, \boldsymbol{\theta})=i \frac{2 m}{\hbar} \partial_{s} \Psi(x, s, \boldsymbol{\theta}) .
$$

More precisely, the stationary wave $\widehat{u}(x, \lambda, \boldsymbol{\theta})$ equals the spatial part $\Psi(x, 0, \boldsymbol{\theta}, \lambda)$ of the solution $\Psi(x, s, \boldsymbol{\theta}, \lambda)=\Psi(x, 0, \boldsymbol{\theta}, \lambda) e^{-i \lambda s / \hbar}$ with energy $E=\lambda^{2}$ and the general solution is a superposition of these waves. Singularities of a scattered wave in the time domain appear in the frequency domain as slower decay of the Fourier transform in directions that belong to the wave front set. The leading singularity of $u_{s c}(x, t, \boldsymbol{\theta})$ in time corresponds to the high frequency asymptote of $\widehat{u}_{s c}(x, \lambda, \boldsymbol{\theta})=\Psi(x, 0, \boldsymbol{\theta}, \lambda)-\widehat{u}_{I}(x, \lambda, \boldsymbol{\theta})$, that is, the first term of an asymptotic series expansion with respect to the variable $\lambda$. The empirical correlations for a potential in $C_{c}^{\infty}\left(\mathbb{R}^{n}\right)$ with the support almost surely contained in a ball $B(0, R)$ is the time domain counterpart of

$$
\mathbb{E}\left(c_{k, n} \prod_{j=1}^{k} \lim _{\lambda \rightarrow \infty} e^{-i \lambda x_{j} \cdot \theta_{j}} \lambda \Psi_{s c}\left(x_{j}, 0, \boldsymbol{\theta}, \lambda\right)\right), \quad \mathbf{x} \in \prod_{j=1}^{k} \mathbb{R}^{n} \backslash \overline{B(0, R)}, \quad \lambda \in \mathbb{R} \backslash\{0\}
$$

which can be obtained directly from the progressive wave expansion [2]. The empirical correlations therefore describes statistical correlations in scattering patterns produced by multiple high-energy particles interacting with the random potential. Similar interpretation is expected to be valid for potentials with less regularity but will not be studied here.

The empirical correlations are invariant with respect to shifts along the trajectories:

$$
D^{k}(\mathbf{x}, \boldsymbol{\theta})=D^{k}\left(x_{1}+s_{1} \theta_{1}, \ldots, x_{k}+s_{k} \theta_{k}, \boldsymbol{\theta}\right), s_{1}, \ldots, s_{k} \in \mathbb{R}
$$

Therefore, without loss of information, it can alternatively be given on the tangent bundle $\prod_{j=1}^{k} T \mathbb{S}^{n-1}$ by identifying each fiber $T_{\theta} \mathbb{S}^{n-1}$, with the orthogonal complement $\{\theta\}^{\perp} \times\{\theta\} \subset$ $\mathbb{R}^{n} \times\{\theta\}=T_{\theta} \mathbb{R}^{n},\{\theta\}^{\perp}:=\left\{x \in \mathbb{R}^{n}: x \cdot \theta=0\right\}$ (see Appendix). The empirical correlations are then expressed as a generalised function

$$
\boldsymbol{\theta} \mapsto D^{k}(\boldsymbol{\theta}) \in \mathcal{D}^{\prime}\left(T_{\boldsymbol{\theta}} \prod_{j=1}^{k} \mathbb{S}^{n-1}\right), \boldsymbol{\theta} \in \prod_{j=1}^{k} \mathbb{S}^{n-1},
$$

given by

$$
D^{k}(\boldsymbol{\theta})\left(y_{1}, \ldots, y_{k}\right)=D^{k}\left(\left(y_{1}, 0\right), \ldots,\left(y_{k}, 0\right), \boldsymbol{\theta}\right)
$$

in coordinates $y_{j}=\left(y_{j}^{1}, \ldots, y_{j}^{n-1}\right) \in \mathbb{R}^{n-1}$ of $T_{\theta_{j}} \mathbb{S}^{n-1}, j=1, \ldots, k$. For a smooth potential one obtains more practical form, $D^{k} \in C^{\infty}\left(\prod_{j=1}^{k} T \mathbb{S}^{n-1}\right)$ which is applied in Proposition 2.1. In comparison with the typical far-field measurement the empirical correlations contain additional tangential parameters $\left(y_{1}, \ldots, y_{k}\right)$. As the name suggests, $D^{k}$ models measurements of correlation between amplitude peaks of leading singularities which scatter from randomly varying objects as a result of interaction between the associated potential and the incident waves. We emphasise that correlations are collected for superpositions of incoming plane waves in all possible incoming directions and the data is collected in the forward directions of the waves. A tangential parameter $y_{j}, j \in\{1, \ldots, k\}$ corresponds to a point on the surface of a detector plate or a film, located on the opposite side of the object in the direction $\theta_{j}$. 
In order to perform the measurement experimentally there is a need for a numerical interpretation of the element $D^{k}$. Perhaps the most practical setup is to sample a system associated with a potential that evolves randomly in time. The interpretation requires a separation of scales regime between the time taken and the size of random variations produced as a wave travel through the medium. A random state of the target has to vary slowly compared to either the speed of propagation of waves (e.g. the speed of light or sound) or the inverse of the radius of target. To state this more accurately, we recall that in the context of physics the wave equation $(\square+V(x)) u(x, t)=0$ is obtained from another wave equation,

$$
\left(\partial_{t}-c^{2} \Delta+V(x)\right) u(x, t)=0,
$$

with wave speed $c>0$ by a certain choice of units. The travel time for a wave that propagates through the target located in the ball $B(0, R)$ of radius $R>0$ is at the most $\frac{2 R}{c}$. Let $\tau$ be the time required for the potential to vary into a new random state that is sufficiently weakly correlated with the previous one. The separation of scales above refers to

$$
\tau \gg \frac{2 R}{c} .
$$

In contrast to operating $k$ detectors simultaneously, slow variation would also make it relevant to apply different separation of scales to reduce the size of a measurement apparatus needed if a single detector could be relocated relatively fast to capture images of one realisation of the potential in several different angles. In that case,

$$
\tau \gg k \frac{2 R}{c}+k T_{0},
$$

where $T_{0}$ is the time required to change the measurement configuration. To compute expectations from discrete measurements one needs more prior information, for example, the ergodicity assumption: Suppose there exists an ergodic transformation $F$ describing iteratively how given potential evolves between the sampling times. Then it is a consequence of the pointwise ergodic theorem that $D^{k}$ is obtained as the average of the samples, i.e.,

$$
D^{k}(\mathbf{x}, \boldsymbol{\theta})=\lim _{m \rightarrow \infty} \frac{1}{m} \sum_{l=0}^{m-1}\left(\lim _{s \rightarrow \infty} \prod_{j=1}^{k}\left[u\left(F^{l} V\right)_{s c}\right]_{\Sigma_{j}}\left(x_{j}+s \theta_{j}, \boldsymbol{\theta}\right)\right)
$$

for almost every potential $V$. The element $u\left(F^{l} V\right)$ above stands for the wave (1) interacting with the potential $x \mapsto F^{l} V(x)$. The limit is taken in the space of generalised functions. The proof falls outside the context of this paper.

1.1. The Results. For a random field

$$
V: \mathbb{R}^{n} \times \Omega \rightarrow \mathbb{R}:(x, \omega) \mapsto V(x, \omega)
$$

we introduce the following three conditions:

(C1) $V \in H^{2}\left(\mathbb{R}^{n}\right) \cap L^{\infty}\left(\mathbb{R}^{n}\right)$ almost surely

(C2) There is a compact set $K \subset \mathbb{R}^{n}$ such that $V$ is supported in $K$ almost surely

(C3) There is a constant $a>0$ such that $\mathbb{E} e^{a\|V\|_{H^{2}}}<\infty$,

The compact set $K$ in (C2) can be replaced by a ball. A field with almost surely bounded $H^{2}$-norm, $\|V\|_{H^{2}} \in L^{\infty}(\Omega)$, satisfies (C3).

Our main results are given by the following theorems. 
Theorem 1.1. Let $V: \mathbb{R}^{n} \times \Omega \rightarrow \mathbb{R}$ be a random field that satisfies the conditions (C1) and (C2) above. Then the $k^{\text {th }}$ moment map $M^{k} \in \mathcal{E}^{\prime}\left(\prod_{j=1}^{k} \mathbb{R}^{n}\right)$, given by

$$
M^{k}(\mathbf{x})=\mathbb{E}\left(\prod_{j=1}^{k} V\left(x_{j}\right)\right),
$$

is uniquely determined by the empirical correlations (2) for any $k \in \mathbb{N}$.

In particular, the proof of Theorem 1.1 provides a reconstruction strategy to recover an explicitly defined sequence $M_{\epsilon}^{k}, \epsilon>0$ of smooth functions that converges to $M^{k}$. Moreover, as Gaussian random fields are determined by their mean field and covariance function, the Theorem 1.1 yields an immediate corollary for this important class of random models.

Corollary 1.1. If the potential $V$ in Theorem 1.1 is a Gaussian random field, then the probability distribution of $V$ is uniquely determined given the empirical correlations

$$
(\mathbf{x}, \boldsymbol{\theta}) \mapsto D^{2}(\mathbf{x}, \boldsymbol{\theta}), \quad(\mathbf{x}, \boldsymbol{\theta}) \in\left(\Pi_{j=1}^{2}\left(\mathbb{R}^{n} \times \mathbb{S}^{n}\right)\right) \backslash \Xi .
$$

Theorem 1.1 can be applied to derive sufficient conditions for the associated laws $V_{*} \mathbb{P}$ : $A \mapsto \mathbb{P}\{\omega \in \Omega: V(\cdot, \omega) \in A\}$ in $H^{2}\left(\mathbb{R}^{n}\right)$ to be unique, i.e., the only positive Borel measure related to the empirical correlations:

Theorem 1.2. Let $V$ and $W$ be two random fields that satisfy the conditions $(\mathrm{C} 1),(\mathrm{C} 2)$, (C3) and yield the same empirical correlations for every $k \in \mathbb{N}$. Then the potentials have the same laws (i.e. probability distributions):

$$
V_{*} \mathbb{P}=W_{*} \mathbb{P} .
$$

The proof of Theorem 1.2 relies partly on a result from [3] considering determinateness for Euclidean multivariate moment problem. This is the main reason to consider fields of the form (C3).

1.2. Future Work. One could also consider an analogous problem for a wave equation with a random index of refraction which is more natural model for some applications. The extension leads to potential scattering in Riemannian geometry where the metric is a random variable. Such a result for sufficiently general class of geometries is one of our long-term objectives. The biggest challenge in that case is randomness of the geodesics that describe trajectories of the wave fronts. The approach in our paper is strongly based on invariance of the trajectories and therefore can not be directly generalised without prior knowledge of the bicharacteristics. Assuming that there is a known average geodesic (e.g. a straight line) for every initial vector would probably be useful. Even in that case one would have to deal with caustics, randomly occuring in a singular support of a wave, thus making it difficult to recover the associated geodesic ray transform from singularities.

1.3. Previous Literature. A natural path for acquiring the spatial correlation data in practise is averaging a large number of independent observations of the scattered field in time. Similar problem setup often appears in wave and particle propagation in heterogeneous media. Typically, heterogeneous medium is modelled as a realization of random field with a priori known statistics. In literature, multi-scale analysis or homogenization is often utilized with the aim of capturing the effective properties of the propagation. We refer to the articles 
$[4,5,6,7,8]$ for various perspectives on wave propagation (whether classical or quantum) in random media.

The origin of the randomness can also be a specific source in the considered system, see e.g. the early work [9] on inverse random source problems. Since then correlation based imaging in random source problems have been considered widely in the framework of different PDE models by Li, Bao and others $[10,11,12,13,14,15]$. Other applications include telescope imaging [16] and seismic imaging [17, 1, 18, 19, 20]. Imaging in random media has also been studied by Borcea and others [21, 22, 23, 24], and for backscattering by Shevtsov [25].

Our paper provides continuation to previous work by the authors in $[26,27,28]$, where the averaging procedure to estimate correlations is based on a single realization of the observed data, i.e., the random potential or boundary condition is sampled only once. Such an approach can reveal valuable information of the leading order statistics of the unknown field. However, the full probability distribution of the unknown is not recovered unlike here.

The literature on inverse scattering for deterministic potentials is rather wide and we cite here only a few works in the field. In [29], Colton and Kirsch introduced the linear sampling method to determine the support of an imperfect conductor given the far-filed of the scattered wave. Uniqueness for the inverse acoustic medium problem was proved by Nachman [30], Novikov [31], and Ramm [32]. Uniqueness for the inverse backscattering problem in a generic class of potentials was proved by Eskin and Ralston [33, 34]. Uniqueness for angularly controlled potentials has been proved by Rakesh and Uhlmann [2]. Single measurement inverse problems for the wave equation is explored by Rakesh [35] and by Liu and others [36, 37]. Uniqueness of a potential for the Neumann-to-Dirichlet type boundary value problem is studied by Rakesh and Symes [38]. Use of moments in inverse problems for partial differential equations has previously been studied by Kurylev and others in [39, 40, 41].

Finally, we want to point out that there exists a variety of criteria for the moment problem to be determinate. These might provide potential alternatives for applications, where the exponential moment is not bounded. This aspect is not the focus of our work and we refer to [42] and references therein regarding such generalizations.

\section{Proofs of the Results}

2.1. Preliminary Definitions. Let us introduce some relevant notations and definitions. Given a distribution $v \in \mathcal{D}^{\prime}(X)$ on a smooth manifold $X$ let $W F(v)$ be the wave front set of $v$, i.e., the complement of the collection of co-vectors $\left(z_{0}, \xi_{0}\right) \in X \times\left(\mathbb{R}^{\operatorname{dim}(X)} \backslash\{0\}\right)$ such that in some neighbourhoods $U \ni z_{0}$ and $V \ni \xi_{0}$ the decay estimate

$$
\widehat{\varphi v}(\tau \xi)=O\left(\tau^{-m}\right) \text {, for } \tau \rightarrow \infty \text {, uniformly in } \xi \in V,
$$

holds for every $\varphi \in C_{c}^{\infty}(U)$ and $m \in \mathbb{N}$. Let $\Gamma$ be a closed cone in $T^{*}(X)$ and define $\mathcal{D}_{\Gamma}^{\prime}(X)$ as the collection of distributions $v \in \mathcal{D}^{\prime}(X)$ such that $W F(v) \subset \Gamma$. Similarly, we define

$$
H_{\Gamma, l o c}^{s}(X):=H_{l o c}^{s}(X) \cap \mathcal{D}_{\Gamma}^{\prime}(X)
$$

for $s \in \mathbb{R}$. Given a submanifold $Y \subset X$ we denote by $N^{*} Y$ the conormal bundle of $Y$, that is, the collection of vectors $\xi \in T^{*} X$ such that $\langle\xi, v\rangle=0$ for every $v \in T_{\pi(\xi)} Y$, where $\pi: T^{*} X \rightarrow X$ stands for the bundle projection. Given a distribution $v$ on $\mathbb{R}^{n+1}$ with $W F(v) \cap N^{*} \Sigma_{i}=\emptyset$, the trace $\operatorname{Tr}_{\Sigma_{i}}(v) \in \mathcal{D}^{\prime}\left(\Sigma_{i}\right)$ is well defined and depends continuously on $v$ with respect to the topology of distributions (see [43]). 
It is shown in the next section that $u_{s c}$ can be split into two parts $u_{s c}=\widetilde{u}_{s c}+u_{R}$ where

$$
\widetilde{u}_{s c}(\cdot, \cdot, \boldsymbol{\theta}) \in H_{\Gamma, l o c}^{-1}\left(\left(\mathbb{R}^{n} \backslash \overline{B(0, R)}\right) \times \mathbb{R}\right),
$$

with

$$
\Gamma:=\bigcup_{j=1}^{k} N^{*} \Sigma_{j} \cup\left\{(x, t ; \xi, k) \in T^{*} \mathbb{R}^{n+1}:(x, t) \in \mathbb{R}^{n+1},\left\langle\xi, \theta_{j}\right\rangle=0, k=0\right\},
$$

and $u_{R} \in H_{l o c}^{1}\left(\mathbb{R}^{n+1}\right)$ for compactly supported $V \in H^{2}\left(\mathbb{R}^{n}\right) \cap L^{\infty}\left(\mathbb{R}^{n}\right)$. Given $\theta_{i} \in \mathbb{S}^{n-1}$, we are interested in discontinuity across $\Sigma_{i}$ which appears in the first term of the decomposition. Amplitude of the peak is defined by the difference

$$
\left[u_{s c}\right]_{\Sigma_{i}}(x, \boldsymbol{\theta}):=\left(\operatorname{Tr}_{\Sigma_{i}}^{+}-\operatorname{Tr}_{\Sigma_{i}}^{-}\right) \widetilde{u}_{s c}\left(x, x \cdot \theta_{i}, \boldsymbol{\theta}\right)
$$

between the two limits $\operatorname{Tr}_{\Sigma_{i}}^{ \pm} \widetilde{u}_{s c}:=\lim _{\epsilon \rightarrow 0 \pm} \operatorname{Tr}_{\Sigma_{i}} \circ S_{\epsilon}^{*}$, where

$$
\begin{gathered}
S_{\epsilon}^{*}: H_{\Gamma, l o c}^{-1}\left(\left(\mathbb{R}^{n} \backslash \overline{B(0, R)}\right) \times \mathbb{R}\right) \rightarrow H_{\Gamma_{\epsilon}, l o c}^{-1}\left(\left(\mathbb{R}^{n} \backslash \overline{B(0, R)}\right) \times \mathbb{R}\right), \\
\Gamma_{\epsilon}:=\left\{(x, t-\epsilon ; v) \in T^{*} \mathbb{R}^{n+1}:(x, t ; v) \in \Gamma\right\}
\end{gathered}
$$

is the pull-back generated by $S_{\epsilon}:(x, t) \mapsto(x, t+\epsilon)$. As shown in Section 2.3, the limits exist for compactly supported potentials in $H^{2}\left(\mathbb{R}^{n}\right) \cap L^{\infty}\left(\mathbb{R}^{n}\right)$. It is a consequence of the trace theorem that the amplitude $\left[u_{s c}\right]$ does not depend on the choice of the decomposition

$$
u_{s c}=\widetilde{u}_{s c}+u_{R} \in H_{\Gamma, l o c}^{-1}\left(\left(\mathbb{R}^{n} \backslash \overline{B(0, R)}\right) \times \mathbb{R}\right)+H_{l o c}^{1}\left(\mathbb{R}^{n+1}\right) .
$$

In particular, the formal notation $\left(\operatorname{Tr}_{\Sigma_{i}}^{+}-\operatorname{Tr}_{\Sigma_{i}}^{-}\right) u_{s c}\left(x, x \cdot \theta_{i}, \boldsymbol{\theta}\right)$ in the introduction makes sense.

2.2. Unique recovery for smooth potentials. Here we prove that arbitrary moments of the random potential are uniquely recovered by the empirical correlations if we know a priori that the potential is smooth. This partial result is the basis for the full proof of Theorem 1.1 in Section 2.3.

Proposition 2.1. Let $V: \mathbb{R}^{n} \times \Omega \rightarrow \mathbb{R}$ be a random field that such that the condition (C2) holds and $V \in C^{\infty}\left(\mathbb{R}^{n}\right)$ almost surely. Then $M^{k} \in \mathcal{E}^{\prime}\left(\prod_{j=1}^{k} \mathbb{R}^{n}\right)$, given by $(4)$, is uniquely determined by the correlations (2) for any $k \in \mathbb{N}$.

Proof. By linearity and uniqueness of the solution the scattered wave is of the form

$$
u_{s c}(x, t, \boldsymbol{\theta})=\sum_{j=1}^{N} v_{s c}\left(x, t, \theta_{j}\right),
$$

where $v_{s c}(x, t, \theta)$ is the scattered part of $v(x, t, \theta)$, defined by

$$
\begin{aligned}
(\square-V(x)) v(x, t, \theta) & =0, \\
v(x, t, \theta) & =\delta(t-x \cdot \theta)+v_{s c}(x, t, \theta), \\
v_{s c}(x, t, \theta) & =0, \quad \text { for } t=-\operatorname{diam}(K),
\end{aligned}
$$

for $(x, t, \theta) \in \mathbb{R}^{n+1} \times \mathbb{S}^{n-1}$. Let $a_{\alpha}(x, \theta) \in C^{\infty}\left(\mathbb{R}^{n} \times \mathbb{S}^{n-1}\right), \alpha \in \mathbb{N} \backslash\{0\}$, be the coefficients of the following asymptotic expansion:

$$
v_{s c}(x, t, \theta)=\frac{1}{2} a_{1}(x, \theta) H(t-x \cdot \theta)+\frac{1}{2} \sum_{\alpha=1}^{s} a_{\alpha+1}(x, \theta)(t-x \cdot \theta)_{+}^{\alpha} \bmod C^{s+2},
$$


where the identity is up to functions in $C^{s+2}\left(\mathbb{R}^{n+1}\right)$ and $H$ is the Heaviside step function,

$$
H(x)=\left\{\begin{array}{ll}
1, & \text { for } x \geq 0 \\
0, & \text { for } x<0
\end{array} .\right.
$$

We recall from $[2,44,45]$ that for a smooth compactly supported potential the expansion exists and is given recursively by

$$
\alpha \theta \cdot \nabla a_{\alpha+1}(x, \theta)=\frac{1}{2}(\Delta+V(x)) a_{\alpha}(x, \theta), \alpha=1,2,3, \ldots
$$

and

$$
\theta \cdot \nabla a_{1}(x, \theta)=V(x) .
$$

Due to the zero initial value of the scattered wave, the first coefficient equals the line integral:

$$
a_{1}(x, \theta)=\int_{-\infty}^{0} V(x+s \theta) d s .
$$

Therefore, we have the identity

$$
\lim _{s \rightarrow \infty} a_{1}(x+s \eta, \theta)=\left\{\begin{array}{ll}
0, & \eta \neq \theta \\
\int_{\mathbb{R}} V(x+s \theta) d s, & \eta=\theta .
\end{array},\right.
$$

for $\eta \in \mathbb{S}^{n-1}$. A restriction of $v_{s c}(x, t, \theta)$ to the boundary $t=x \cdot \theta$ at any distant point along the ray $x+s \theta, s \in \mathbb{R}$ is simply the associated ray transform of the potential. Applying (7) implies

$$
\left[u_{s c}\right]_{\Sigma_{i}}\left(x+s \theta_{i}, \boldsymbol{\theta}\right)=\frac{1}{2} a_{1}\left(x+s \theta_{i}, \theta_{i}\right)
$$

for a collection of distinct vectors $\theta_{1}, \ldots, \theta_{k}$. Therefore, the empirical correlations at points $(\mathbf{x}, \boldsymbol{\theta}) \in \prod_{j=1}^{k}\left(\mathbb{R}^{n} \times \mathbb{S}^{n-1}\right) \backslash \Xi$ satisfy

$$
\begin{aligned}
D^{k}(\mathbf{x}, \boldsymbol{\theta}) & =\mathbb{E}\left(\lim _{s \rightarrow \infty} \prod_{j=1}^{k}\left[u_{s c}\right]_{\Sigma_{1}}\left(x_{j}+s \theta_{j}, \boldsymbol{\theta}\right)\right) \\
& =\frac{1}{2^{k}} \mathbb{E}\left(\prod_{j=1}^{k} \int_{\mathbb{R}} V\left(x_{j}+s_{j} \theta_{j}\right) d s_{j}\right) \\
& =\frac{1}{2^{k}} \int_{\mathbb{R}^{k}} \mathbb{E}\left(\prod_{j=1}^{k} V\left(x_{j}+s_{j} \theta_{j}\right)\right) d s_{1} \ldots d s_{k} \\
& =\frac{1}{2^{k}} \int_{L(\mathbf{x}, \boldsymbol{\theta})} \mathbb{E}\left(\prod_{j=1}^{k} V\left(z_{j}\right)\right) d l\left(z_{1}, \ldots, z_{k}\right),
\end{aligned}
$$

where $L(\mathbf{x}, \boldsymbol{\theta})$ is the affine subspace

$$
L(\mathbf{x}, \boldsymbol{\theta}):=\left\{\mathbf{x}+\boldsymbol{h} \in \prod_{j=1}^{k} \mathbb{R}^{n}: \boldsymbol{h} \in H_{\boldsymbol{\theta}}\right\},
$$


where

$$
H_{\boldsymbol{\theta}}:=\left\{\left(s_{1} \theta_{1}, \ldots, s_{k} \theta_{k}\right) \in \prod_{j=1}^{k} \mathbb{R}^{n}: s_{1}, \ldots, s_{k} \in \mathbb{R}\right\},
$$

and $d l\left(z_{1}, \ldots, z_{k}\right)$ denotes the pull-back volume form which is induced from the canonical volume form via the inclusion map $L(\mathbf{x}, \boldsymbol{\theta}) \hookrightarrow \prod_{j=1}^{k} \mathbb{R}^{n}$. From the identity (8) one concludes that the empirical correlations for a smooth potential extend by continuity into a smooth function of variables $(\mathbf{x}, \boldsymbol{\theta}) \in \prod_{j=1}^{k}\left(\mathbb{R}^{n} \times \mathbb{S}^{n-1}\right)$.

The main idea of the next step is as follows: Based on the identity (8) and the fact that every $(n k-1)$-dimensional shifted hyperplane in $\prod_{j=1}^{k} \mathbb{R}^{n}$ is obtained by stacking up spaces $L(\mathbf{x}, \boldsymbol{\theta})$ with different parameters $\mathbf{x}, \boldsymbol{\theta}$ we can reconstruct the Radon transform of the $k^{\text {th }}$ moment map, $M^{k}(\mathbf{x}):=\mathbb{E}\left(\prod_{j=1}^{k} V\left(x_{j}\right)\right)$ from the empirical correlations. That is to say, for arbitrary $(r, \boldsymbol{\eta}) \in \mathbb{R} \times \mathbb{S}^{n k-1}$ one divides the hyperplane

$$
\Gamma(r, \boldsymbol{\eta}):=\left\{\boldsymbol{z} \in \prod_{j=1}^{k} \mathbb{R}^{n}: \boldsymbol{z} \cdot \boldsymbol{\eta}=r\right\}
$$

into distinct subspaces of the form $L(\mathbf{x}, \boldsymbol{\theta})$ given in equation (9), then applies the empirical correlations together with (8) to obtain integrals $\int_{L} M^{k} d l$ of the moment map over each of the subspaces and finally computes the superposition of them.

Let us formulate this idea rigorously. First, we provide a representation of the hyperplane $\Gamma(r, \boldsymbol{\eta})$ as an orthogonal decomposition involving the subspace $L$ in (9). Construct arbitrary smooth functions

$$
\theta_{j}: \mathbb{S}^{n k-1} \rightarrow \mathbb{S}^{n-1}
$$

to satisfy

$$
\eta_{j} \cdot \theta_{j}(\boldsymbol{\eta})=0
$$

for any $j=1, \ldots, k$. Next, let $\boldsymbol{\theta}:=\left(\theta_{1}, \ldots, \theta_{k}\right)$ and define the set

$$
P(\boldsymbol{\eta}, \boldsymbol{\theta}) \subset \prod_{j=1}^{k} \mathbb{R}^{n}
$$

to be the maximal linear subspace orthogonal to $\boldsymbol{\eta}$ and vectors

$$
(0, \ldots, 0, \underbrace{\theta_{j}(\boldsymbol{\eta})}_{j^{\text {th }} \text { slot }}, 0, \ldots, 0) \in \prod_{j=1}^{k} \mathbb{R}^{n}, j=1, \ldots, k,
$$

simultaneously. Clearly, each $\mathbf{x} \in \Gamma(r, \boldsymbol{\eta})$ is uniquely written in form $\mathbf{x}=\mathbf{x}^{L}+\mathbf{x}^{P}$, where

$$
\mathbf{x}^{L}=\left(x_{1}^{L}, \ldots, x_{k}^{L}\right) \in L(r \boldsymbol{\eta}, \boldsymbol{\theta}):=L\left(r \eta_{1}, \ldots, r \eta_{k}, \boldsymbol{\theta}\right),
$$

$x_{j}^{L} \in \mathbb{R}^{n}$ for every $j=1, \ldots, k$, and

$$
\mathbf{x}^{P}=\left(x_{1}^{P}, \ldots, x_{k}^{P}\right) \in P(\boldsymbol{\eta}, \boldsymbol{\theta}),
$$

where $x_{j}^{P} \in \mathbb{R}^{n}$ for $j=1, \ldots, k$. In consequence, we have that

$$
\Gamma(r, \boldsymbol{\eta})=L(r \boldsymbol{\eta}, \boldsymbol{\theta}) \oplus P(\boldsymbol{\eta}, \boldsymbol{\theta}) .
$$


Now, by identity (8), the Radon transform of the moment function $M^{k}$ at $(r, \boldsymbol{\eta}) \in \mathbb{R} \times \mathbb{S}^{n k-1}$ takes the form

$$
\begin{aligned}
R\left[M^{k}\right](r, \boldsymbol{\eta}) & =\int_{\Gamma(r, \boldsymbol{\eta})} M^{k}(\mathbf{x}) d \nu(\mathbf{x}) \\
& =\int_{P(\boldsymbol{\eta}, \boldsymbol{\theta})} \int_{L(r \boldsymbol{\eta}, \boldsymbol{\theta})} \mathbb{E}\left(\prod_{j=1}^{k} V\left(x_{j}^{L}+x_{j}^{P}\right)\right) d l\left(\mathbf{x}^{L}\right) d P\left(\mathbf{x}^{P}\right) \\
& =\int_{P(\boldsymbol{\eta}, \boldsymbol{\theta})} \int_{L\left(r \boldsymbol{\eta}+\mathbf{x}^{P}, \boldsymbol{\theta}\right)} \mathbb{E}\left(\prod_{j=1}^{k} V\left(z_{j}\right)\right) d l(\boldsymbol{z}) d P\left(\mathbf{x}^{P}\right) \\
& =2^{k} \int_{P(\boldsymbol{\eta}, \boldsymbol{\theta})} D^{k}\left(r \boldsymbol{\eta}+\mathbf{x}^{P}, \boldsymbol{\theta}(\boldsymbol{\eta})\right) d P\left(\mathbf{x}^{P}\right),
\end{aligned}
$$

where $d P, d l$, and $d \nu$ are the canonical volume forms, induced by inclusions into $\prod_{j=1}^{k} \mathbb{R}^{n}$. Let $\iota_{\boldsymbol{\theta}(\boldsymbol{\eta})}: T_{\boldsymbol{\theta}(\boldsymbol{\eta})} \prod_{j=1}^{k} \mathbb{S}^{n-1} \hookrightarrow T \prod_{j=1}^{k} \mathbb{S}^{n-1}$ be the trivial inclusion. Considering the empirical correlations as a function $D^{k} \in C_{c}^{\infty}\left(T \prod_{j=1}^{k} \mathbb{S}^{n-1}\right)$ reduces (11) to

$$
R\left[M^{k}\right](r, \boldsymbol{\eta})=\left\langle\delta\left(r-\boldsymbol{\eta} \cdot \iota_{\boldsymbol{\theta}(\boldsymbol{\eta})}(\boldsymbol{v})\right), D^{k} \circ \iota_{\boldsymbol{\theta}(\boldsymbol{\eta})}(\boldsymbol{v})\right\rangle=\left\langle\left(\iota_{\boldsymbol{\theta}(\boldsymbol{\eta})}\right)_{*} \delta\left(r-\boldsymbol{\eta} \cdot \iota_{\boldsymbol{\theta}(\boldsymbol{\eta})}\right), D^{k}\right\rangle,
$$

where we denote $\boldsymbol{v} \in T_{\boldsymbol{\theta}(\boldsymbol{\eta})} \prod_{j=1}^{k} \mathbb{S}^{n-1}$ and identify $T_{\theta} \mathbb{S}^{n-1}=\{\theta\}^{\perp} \times\{\theta\} \subset \mathbb{R}^{n} \times \mathbb{R}^{n}$. Finally, to obtain $M^{k}$ from the transformed quantity one applies the Radon inversion formula,

$$
\Delta^{(n k-1) / 2} R^{*} R=i d,
$$

where $R^{*}$ is the adjoint of the Radon transform and

$$
\Delta^{(n k-1) / 2} f(x):=\int_{\mathbb{R}^{n k}} e^{i x \cdot \xi}|\xi|^{n k-1} \widehat{f}(\xi) d \xi .
$$

More precisely, by (12), the Radon inversion formula implies

$$
\Psi^{k}\left\{D^{k}\right\}(\mathbf{x})=M^{k}(\mathbf{x})
$$

where

$$
\Psi^{k}:=\Delta^{(n-1) / 2} R^{t} A^{k}
$$

and

$$
A^{k}: C_{c}^{\infty}\left(\prod_{j=1}^{k} T \mathbb{S}^{n-1}\right) \rightarrow C^{\infty}\left(\mathbb{R} \times \mathbb{S}^{k n-1}\right), A^{k}(r, \boldsymbol{\eta}):=\left(\iota_{\boldsymbol{\theta}(\boldsymbol{\eta})}\right)_{*} \delta\left(r-\boldsymbol{\eta} \cdot \iota_{\boldsymbol{\theta}(\boldsymbol{\eta})}\right) .
$$

This concludes the proof.

We will refer to the map $\Psi^{k}$ as a reconstruction operator for smooth potentials.

Remark 2.1. It is well known that theory of Fourier integral operators can be applied to study stability of reconstruction from the X-ray transform. The function

$$
\iota_{\boldsymbol{\theta}(\cdot)}: \boldsymbol{\theta}^{*} T \prod_{j=1}^{k} \mathbb{S}^{n-1} \rightarrow T \prod_{j=1}^{k} \mathbb{S}^{n-1}, \quad \iota_{\boldsymbol{\theta}(\cdot)}(\boldsymbol{\eta}, \boldsymbol{v}):=(\boldsymbol{\theta}(\boldsymbol{\eta}), \boldsymbol{v}),
$$


defined on the pull-back bundle $\boldsymbol{\theta}^{*} T \prod_{j=1}^{k} \mathbb{S}^{n-1}$, generates the Fourier integral operator

$$
\iota_{\boldsymbol{\theta}(\cdot)}^{*}: C_{c}^{\infty}\left(\prod_{j=1}^{k} T \mathbb{S}^{n-1}\right) \rightarrow \mathcal{D}^{\prime}\left(\boldsymbol{\theta}^{*} T \prod_{j=1}^{k} \mathbb{S}^{n-1}\right) .
$$

Composition of the operator with another Fourier integral operator,

$$
K: C_{c}^{\infty}\left(\boldsymbol{\theta}^{*} T \prod_{j=1}^{k} \mathbb{S}^{n-1}\right) \rightarrow \mathcal{D}^{\prime}\left(\mathbb{R} \times \mathbb{S}^{n k-1}\right)
$$

defined by the kernel

$$
K(r, \boldsymbol{\eta} ; \widetilde{\boldsymbol{\eta}}, \widetilde{\boldsymbol{v}}):=\delta(\widetilde{\boldsymbol{\eta}}-\boldsymbol{\eta}) \delta\left(r-\boldsymbol{\eta} \cdot \iota_{\boldsymbol{\theta}(\boldsymbol{\eta})}(\widetilde{\boldsymbol{v}})\right), \quad(r, \boldsymbol{\eta} ; \widetilde{\boldsymbol{\eta}}, \widetilde{\boldsymbol{v}}) \in \mathbb{R} \times \mathbb{S}^{n k-1} \times \boldsymbol{\theta}^{*} T \prod_{j=1}^{k} \mathbb{S}^{n-1},
$$

agrees with $A^{k}$. Consequently, the reconstruction operator $\Psi^{k}$ is a composition of known Fourier integral operators whose stability properties in Sobolev spaces are stated in [43, Corollary 4.4.5]. A composition of the canonical relations is connected to dynamics of instabilities and shapes of artifacts, while the order of regularity is encoded into decay properties of the symbols.

Remark 2.2. One can consider the problem of recovering the moments from a smaller set of measurements. As shown above, the empirical correlations reduce to integration of the moments over certain planar surfaces which the Radon transform is recovered from. For uniqueness of the inversion it is sufficient to reconstruct the Radon transform on the limited domain

$$
\left\{(r, \boldsymbol{\eta}) \in \mathbb{R} \times \mathbb{S}^{n k-1}:|r \boldsymbol{\eta} \cdot \boldsymbol{a}|<\varepsilon\right\}
$$

for fixed $\varepsilon>0$ and $\boldsymbol{a} \in S^{n k-1}$, thus requiring a smaller domain of parameters $(\mathbf{x}, \boldsymbol{\theta})$. This is a consequence of the identity $R\left[M^{k}\right](r, \boldsymbol{\eta})=\widehat{M^{k}}(r \boldsymbol{\eta})$ and uniqueness of continuation for real analytic functions, such as Fourier transforms of compactly supported smooth functions. We refer to [46] and [47] for more studies on the Radon transform.

2.3. Proof of Theorem 1.1, The General Setting. We shall first study the system for a single random parameter $\omega_{0} \in \Omega$. Fix the potential $V(x)=V\left(x, \omega_{0}\right) \in L^{\infty}\left(\mathbb{R}^{n}\right) \cap H^{2}\left(\mathbb{R}^{n}\right)$ that is supported in $B(0, R), R>0$ and write the solution of (1) with a possibly non-smooth potential in the form

$$
u_{s c}(x, t, \boldsymbol{\theta})=\sum_{j=1}^{k} \frac{1}{2} a_{1}\left(x, \theta_{j}\right) H\left(t-x \cdot \theta_{j}\right)+u_{R}(x, t, \boldsymbol{\theta}),
$$

where

$$
a_{1}\left(x, \theta_{j}\right):=\int_{-\infty}^{0} V\left(x+s \theta_{j}\right) d s
$$

and $u_{R}(x, t, \boldsymbol{\theta})$ is the residual term. We will need the following lemmas:

Lemma 2.1. Let $V(x) \in L^{\infty}\left(\mathbb{R}^{n}\right) \cap H^{2}\left(\mathbb{R}^{n}\right)$ be supported in $B(0, R)$. It holds that $x \mapsto$ $H(t-x \cdot \theta) \Delta a_{1}(x, \theta) \in L^{2}\left(\mathbb{R}^{n}\right)$. 
Proof. Consider $\Delta a_{1}(x, \theta)$ as a linear functional

$$
\Delta a_{1}(\cdot, \theta): L^{2}\left(\mathbb{R}^{n}\right) \rightarrow \mathbb{R}: \phi(x) \mapsto \int_{-\infty}^{0} \int_{\mathbb{R}^{n}} \Delta V(x+s \theta) \phi(x) d x d s .
$$

It follows by the Cauchy-Schwarz inequality that

$$
\begin{aligned}
\left|\left\langle H(t-x \cdot \theta) \Delta a_{1}(x, \theta), \phi(x)\right\rangle\right| & =\left|\int_{-\infty}^{0} \int_{\mathbb{R}^{n}} \Delta V(x+s \theta) \phi(x) H(t-x \cdot \theta) d x d s\right| \\
& \leq \int_{-t-2 R \mathbb{R}^{n}}^{0}|\Delta V(x+s \theta) \| \phi(x)| d x d s \\
& \leq \int_{-t-2 R}^{0}\|\Delta V(\cdot+s \theta)\|_{L^{2}\left(\mathbb{R}^{n}\right)}\|\phi\|_{L^{2}\left(\mathbb{R}^{n}\right)} d s \\
& =(2 R+t)\|\Delta V\|_{L^{2}\left(\mathbb{R}^{n}\right)}\|\phi\|_{L^{2}\left(\mathbb{R}^{n}\right)},
\end{aligned}
$$

that is, the functional $H(t-x \cdot \theta) \Delta a_{1}(x, \theta): L^{2}\left(\mathbb{R}^{n}\right) \rightarrow \mathbb{R}$ is bounded. The claim follows by duality.

Lemma 2.2. The residual term $u_{R}$ in (14) satisfies $u_{R}(\cdot, t, \boldsymbol{\theta}) \in H_{l o c}^{1}\left(\mathbb{R}^{n+1}\right)$.

Proof. Let $T>R$. Substituting the ansatz (14) into (1) yields

$$
(\square-V(x)) u_{R}(x, t, \boldsymbol{\theta})=\frac{1}{2} \sum_{j=1}^{k} H\left(t-x \cdot \theta_{j}\right)(V(x)+\Delta) a_{1}\left(x, \theta_{j}\right) .
$$

The idea is to construct a sufficiently regular solution of the equation (15) by extending a local solution that satisfy initial and boundary conditions of the residual. The right side of (15) is a sum of $L^{2}$-functions supported in $B(0, R+t)$ for each $t$. In particular,

$$
(\square-V(x)) w_{R}(x, t, \boldsymbol{\theta}) \in L^{1}\left([-R, T] ; L^{2}(B(0, r))\right)
$$

where $w_{R}$ refers to a solution of $(15)$ in a smaller set $(x, t) \in B(0, r) \times[-R, T]$, with sufficiently large $r>2 R+T$. As no scattering occurs before the incident wave hits the potential, we consider the initial conditions

$$
\begin{cases}w_{R}(x, t, \boldsymbol{\theta})=u_{R}(x, t, \boldsymbol{\theta})=0, & t=-R \\ \partial_{t} w_{R}(x, t, \boldsymbol{\theta})=\partial_{t} u_{R}(x, t, \boldsymbol{\theta})=0, & t=-R .\end{cases}
$$

In $\left(\mathbb{R}^{n} \backslash \overline{B(0, R)}\right) \times \mathbb{R}$ the scattered wave satisfies the free wave equation $\square u_{s c}=0$ so due to the initial values $u_{s c}(x, t, \boldsymbol{\theta})=0=\partial_{t} u_{s c}(x, t, \boldsymbol{\theta}), t<-R$, and unit propagation speed of disturbances the scattered wave is supported within the set

$$
C_{R}:=\left\{(x, t) \in \mathbb{R}^{n+1}: x \in B(0,2 R+t)\right\}
$$

which contains also the support of $a(x, \theta) H(t-x \cdot \theta)$ for each $\theta \in \mathbb{S}^{n-1}$. Consequently, $\operatorname{supp}\left(u_{R}\right) \subset C_{R}$. It is therefore suitable to associate $w_{R}$ with the zero boundary conditions,

$$
w_{R}(x, t, \boldsymbol{\theta})=0, \text { for }(x, t) \in \partial B(0, r) \times[-R, T]
$$


By [48, Ch. IV $\S 3]$ the initial and boundary conditions above ensure existence and uniqueness of $w_{R}(x, t, \boldsymbol{\theta}) \in H^{1}(B(0, r) \times[-R, T])$. Within the space $(B(0, r) \backslash B(0, T+R)) \times[-R, T]$ the function $w_{R}$ satisfies the free wave equation, $\square w_{R}=0$, so applying the energy estimate together with zero initial and boundary conditions one extends the domain of $w_{R}$ into $\mathbb{R}^{n+1}$. This can be seen by first applying the estimate to find a zero extension $\bar{w}_{R} \in H^{1}\left(\mathbb{R}^{n} \times[-R, T]\right)$ of $w_{R}$ with respect to variable $x$ and, second, to extend the domain to the whole timeline $t \in \mathbb{R}$ in the weak sense. Finally, uniqueness of $u_{s c}$ implies that the extension equals $u_{R}$.

Due to regularity of $u_{R}$ the peak $\left[u_{R}\right]_{\Sigma_{i}}$ vanishes. As in the smooth case, the amplitude of the peak singularity carried by the scattered wave reduces to $\frac{1}{2} a_{1}\left(\cdot, \theta_{i}\right)$ on distant regions within the boundary $\Sigma_{i}$ :

$$
\begin{aligned}
{\left[u_{s c}\right]_{\Sigma_{i}}\left(x+s \theta_{i}, \boldsymbol{\theta}\right) } & =\left[\sum_{j=1}^{k} \frac{1}{2} a_{1}\left(x, \theta_{j}\right) H\left(t-x \cdot \theta_{j}\right)\right]_{\Sigma_{i}}\left(x+s \theta_{i}, \boldsymbol{\theta}\right) \\
& =\frac{1}{2} a_{1}\left(x+s \theta_{i}, \theta_{i}\right), \text { where } \theta_{j} \neq \theta_{i}, \text { for } j \neq i, \quad j \in\{1, \ldots, k\} .
\end{aligned}
$$

Consequently, we obtain

$$
\lim _{s \rightarrow \infty}\left[u_{s c}\right]_{\Sigma_{i}}\left(x+s \theta_{i}, \boldsymbol{\theta}\right)=\frac{1}{2} \int_{\mathbb{R}} V\left(x+s \theta_{i}\right) d s
$$

which can be extended to cover the complete set of parameters $\boldsymbol{\theta} \in \prod_{j=1}^{k} \mathbb{S}^{n-1}$.

We shall now apply the previous observations to the probabilistic setting. As earlier, define the empirical correlations for $k$ base points by

$$
D^{k}(\mathbf{x}, \boldsymbol{\theta}):=\mathbb{E}\left(\lim _{s \rightarrow \infty} \prod_{j=1}^{k}\left[u_{s c}\right] \Sigma_{j}\left(x_{j}+s \theta_{j}, \boldsymbol{\theta}\right)\right), \quad(\mathbf{x}, \boldsymbol{\theta}) \in\left(\prod_{j=1}^{k}\left(\mathbb{R}^{n} \times \mathbb{S}^{n-1}\right)\right)
$$

by applying the extension above. For $k=1$, taking convolution with the mollifier $\phi_{\epsilon}(x)=$ $\epsilon^{-n} \phi\left(\epsilon^{-1} x\right), \phi \in C_{c}^{\infty}\left(\mathbb{R}^{n}\right), \int_{\mathbb{R}^{n}} \phi(x) d x=1$ yields

$$
\begin{aligned}
{\left[\phi_{\epsilon} * D^{1}(\cdot, \theta)\right](x) } & =\int_{\mathbb{R}^{n}} \phi_{\epsilon}(z) \mathbb{E}\left(\frac{1}{2} \int_{\mathbb{R}} V(x-z+s \theta) d s\right) d z \\
& =\mathbb{E}\left(\frac{1}{2} \int_{\mathbb{R}} V_{\epsilon}(x+s \theta) d s\right) \\
& =D_{\epsilon}^{1}(x, \theta)
\end{aligned}
$$

where $D_{\epsilon}^{1}(x, \theta)$ is the empirical correlations associated to the mollified smooth potential $V_{\epsilon}(x):=\left(\phi_{\epsilon} * V\right)(x)$. Similarly, one derives

$$
\left[\phi_{\epsilon}^{k} * D^{k}(\cdot, \boldsymbol{\theta})\right](\mathbf{x})=D_{\epsilon}^{k}(\mathbf{x}, \boldsymbol{\theta}),
$$

where $\phi_{\epsilon}^{k}\left(z_{1}, \ldots, z_{k}\right):=\phi_{\epsilon}\left(z_{1}\right) \cdots \phi_{\epsilon}\left(z_{k}\right)$, and $D_{\epsilon}(\mathbf{x}, \boldsymbol{\theta})$ is the empirical correlations (8) at $\mathbf{x} \in \prod_{j=1}^{k} \mathbb{R}^{n}$ for the complete set of directions $\boldsymbol{\theta} \in \prod_{j=1}^{k} \mathbb{S}^{n-1}$ and the smooth potential $V_{\epsilon}$. 
The mollified moment $M_{\epsilon}^{k}$ approximates $M^{k}$ in the sense of generalized functions:

$$
\begin{aligned}
\lim _{\epsilon \rightarrow 0} M_{\epsilon}^{k}(\mathbf{x}) & =\lim _{\epsilon \rightarrow 0} \mathbb{E} \int_{\prod_{j=1}^{k} \mathbb{R}^{n}} \phi_{\epsilon}^{k}\left(x_{1}-z_{1}, \ldots, x_{k}-z_{k}\right) V\left(z_{1}\right) \cdots V\left(z_{k}\right) d z \\
& =\lim _{\epsilon \rightarrow 0} \int_{\prod_{j=1}^{k} \mathbb{R}^{n}} \phi_{\epsilon}^{k}\left(x_{1}-z_{1}, \ldots, x_{k}-z_{k}\right) \mathbb{E}\left(V\left(z_{1}\right) \cdots V\left(z_{k}\right)\right) d z \\
& =\lim _{\epsilon \rightarrow 0} \phi_{\epsilon}^{k} * M^{k}(\mathbf{x}) \\
& =\delta_{0} * M^{k}(\mathbf{x}) \\
& =M^{k}(\mathbf{x}) \in \mathcal{D}^{\prime}\left(\prod_{j=1}^{k} \mathbb{R}^{n}\right)
\end{aligned}
$$

see e.g. [49, Ch 5]. The limit in (20) is to be understood by means of sequential convergence in the topology of generalised functions.

In summary, we obtain a reconstruction strategy which consists of the following three steps:

(Step 1) Regularisation of the empirical correlations: The operator

$$
\Phi^{k}: C^{\infty}\left(\prod_{j=1}^{k} S^{n-1} ; \mathcal{D}^{\prime}\left(\prod_{j=1}^{k} \mathbb{R}^{n}\right)\right) \rightarrow C_{c}^{\infty}\left((0, \infty) \times T \prod_{j=1}^{k} \mathbb{S}^{n-1}\right),
$$

given by

$$
\Phi^{k}(v)(\epsilon, \boldsymbol{y}):=\left[\phi_{\epsilon} * v(\cdot, \boldsymbol{\theta})\right](\boldsymbol{y}), \text { for } \epsilon \in(0, \infty), \boldsymbol{y} \in T_{\boldsymbol{\theta}} \prod_{j=1}^{k} \mathbb{S}^{n-1},
$$

transforms the empirical correlations into a parametrised family of functions which corresponds to the regularised potentials:

$$
\Phi^{k}\left(D^{k}\right)(\epsilon, \boldsymbol{y})=D_{\epsilon}^{k}(\boldsymbol{y}, \boldsymbol{\theta}), \text { for } \boldsymbol{y} \in T_{\boldsymbol{\theta}} \prod_{j=1}^{k} \mathbb{S}^{n-1} .
$$

Above, the regularised correlations are identified with a smooth function on $T \prod_{j=1}^{k} \mathbb{S}^{n-1}$ according to the invariance (3).

(Step 2) Reconstruction of moments from the regularised epirical correlations: Let $\Psi^{k}$ be the reconstruction operator for smooth potentials, given by (13). The associated operator

$$
\tilde{\Psi}^{k}: C_{c}^{\infty}\left((0, \infty) \times T \prod_{j=1}^{k} \mathbb{S}^{n-1}\right) \rightarrow C_{c}^{\infty}\left((0, \infty) \times \prod_{j=1}^{k} \mathbb{R}^{n}\right),
$$

defined by

$$
\tilde{\Psi}^{k} f(\epsilon, \mathbf{x}):=\left[\Psi^{k} f(\epsilon, \cdot)\right](\mathbf{x}), \mathbf{x} \in \prod_{j=1}^{k} \mathbb{R}^{n}, \epsilon \in(0, \infty)
$$

transforms the regularised empirical correlations into the corresponding family of moment maps, i.e.,

$$
\tilde{\Psi}^{k} \Phi^{k} D^{k}(\epsilon, \mathbf{x})=M_{\epsilon}^{k}(\mathbf{x})
$$


(Step 3) High-resolution limit: After the first two steps, the moment map $M^{k} \in \mathcal{E}^{\prime}\left(\prod_{j=1}^{k} \mathbb{R}^{n}\right)$ is obtained by taking the limit $\epsilon \longrightarrow 0$ within the space of generalised functions, that is,

$$
\lim _{\epsilon \rightarrow 0} \Psi^{k} \Phi^{k} D^{k}(\epsilon, \mathbf{x})=M^{k}(\mathbf{x})
$$

Remark 2.3. It is reasonable to believe that there is not any universal numerical scheme to treat the distributional limit $\epsilon \longrightarrow 0$ in general context, that is, without extra regularity conditions for the potential. One could study what requirements for the potential are needed to make the empirical correlations and the moments to lie in a given pair of Sobolev spaces. Provided that such conditions were satisfied, the reconstruction strategy above would consist of a convolution operator on a Sobolev space and the Fourier integral operator $\Psi^{k}$ for which stability properties as a map between Sobolev spaces in $L^{2}$ are known (See Remark 2.1), thus making it more relevant to study numerically. We shall not go into details here since numerical exploration falls out of scope of this paper.

2.4. Proof of Theorem 1.2. Let $\mathbb{R}^{\mathbb{N}}$ stand for the set of infinite sequences $\left(x_{j}\right)_{j=1}^{\infty}, x_{j} \in \mathbb{R}$, $j \in \mathbb{N}$ endowed with the smallest topology for which the coordinate projections are continuous. We recall that $\mathbb{R}^{\mathbb{N}}$ is a Polish space. Also, let $\ell^{2}$ stand for the space of all sequences bounded in the 2-norm. Let $\iota: \ell^{2} \hookrightarrow \mathbb{R}^{\mathbb{N}}$ stand for the trivial inclusion and define

$$
\iota^{-1} \mathcal{B}\left(\mathbb{R}^{\mathbb{N}}\right):=\sigma\left\{\ell^{2} \cap B: B \in \mathcal{B}\left(\mathbb{R}^{\mathbb{N}}\right)\right\} .
$$

Before continuing to the proof we record the following lemma:

Lemma 2.3. The Borel algebra of $\ell^{2}$ is generated by $\iota$, that is, $\mathcal{B}\left(\ell^{2}\right)=\iota^{-1} \mathcal{B}\left(\mathbb{R}^{\mathbb{N}}\right)$.

Proof. Let $\mathcal{F}^{j}=\left(\pi^{\{j\}}\right)^{-1} \mathcal{B}(\mathbb{R})$ be the $\sigma$-algebra generated by the coordinate projection $\pi^{\{j\}}$ : $\mathbb{R}^{\mathbb{N}} \rightarrow \mathbb{R}$, for each $j \in \mathbb{N}$. Since the Borel algebra of $\mathbb{R}$ is generated by open sets, preimages of the form $\left(\pi^{\{j\}}\right)^{-1} U$, where $U \subset \mathbb{R}$ is open, generate $\mathcal{F}^{j}$. As the union $\bigcup_{j \in \mathbb{N}} \mathcal{F}_{j}$ generates $\mathcal{B}\left(\mathbb{R}^{\mathbb{N}}\right)$, the algebra $\iota^{-1} \mathcal{B}\left(\mathbb{R}^{\mathbb{N}}\right)$ is generated by the pre-images, $\iota^{-1}\left(\pi^{\{j\}}\right)^{-1} U, j \in \mathbb{N}$, which are open in $\ell^{2}$ by continuity of the coordinate projections $\left(\pi^{\{j\}} \circ \iota\right), j \in \mathbb{N}$. In particular, $\iota^{-1} \mathcal{B}\left(\mathbb{R}^{\mathbb{N}}\right) \subset \mathcal{B}\left(\ell^{2}\right)$. On the other hand, each closed ball $\bar{B}_{r}$ of $\ell^{2}$ with radius $r>0$, centered at $f \in \ell^{2}$, is the limit set

$$
\bar{B}_{r}=\bigcap_{m=1}^{\infty}\left\{g \in \mathbb{R}^{\mathbb{N}}: \sum_{j=1}^{m}\left|f_{j}-\pi^{\{j\}} g\right|^{2} \in\left[0, r^{2}\right]\right\}, f_{j}:=\pi^{\{j\}} \iota f
$$

which is measurable in $\mathbb{R}^{\mathbb{N}}$ as a countable intersection of measurable sets. Consequently, as every open set of a separable metric space is a countable union of closed balls, the Borel algebra of $\mathbb{R}^{\mathbb{N}}$ contains the topology of $\ell^{2}$ which implies $\mathcal{B}\left(\ell^{2}\right) \subset \iota^{-1} \mathcal{B}\left(\mathbb{R}^{\mathbb{N}}\right)$.

We shall now continue to the proof. Let the random field $V \in H^{2}\left(\mathbb{R}^{n}\right) \cap L^{\infty}\left(\mathbb{R}^{n}\right)$ satisfy

$$
\mathbb{E} e^{a\|V\|_{H^{2}\left(\mathbb{R}^{n}\right)}}<\infty \text {. }
$$

Fix an orthonormal basis $\varphi_{j}, j \in \mathbb{N}$ of $H^{2}\left(\mathbb{R}^{n}\right)$ and let $\mu^{J}\left(V_{j_{1}}, \ldots, V_{j_{k}}\right)$ be the distribution

$$
\mu^{J}\left(V_{j_{1}}, \ldots, V_{j_{k}}\right):=\left(V_{j_{1}}, \ldots, V_{j_{k}}\right)_{*} \mathbb{P}: \mathcal{B}\left(\mathbb{R}^{k}\right) \rightarrow[0,1]
$$

with

$$
\left(V_{j_{1}}, \ldots, V_{j_{k}}\right)_{*} \mathbb{P}(B):=\mathbb{P}\left(\left(V_{j_{1}}, \ldots, V_{j_{k}}\right) \in B\right)
$$


generated by the finite collection of random variables $V_{l}:=\left\langle V, \varphi_{l}\right\rangle, l \in J=\left\{j_{1}, \ldots, j_{k}\right\} \subset \mathbb{N}$. By definition, the moments satisfy

$$
\left\langle M^{k}, \varphi_{j_{1}} \otimes \cdots \otimes \varphi_{j_{k}}\right\rangle=\mathbb{E}\left(\left\langle V, \varphi_{j_{1}}\right\rangle \cdots\left\langle V, \varphi_{j_{k}}\right\rangle\right)=\mathbb{E}\left(V_{j_{1}} \cdots V_{j_{k}}\right)
$$

for every $j_{1}, \ldots, j_{k} \in \mathbb{N}$. These quantities are determined from the empirical correlations by Theorem 1.1. Since,

$$
e^{a\left|\left(V_{j_{1}}, \ldots, V_{j_{k}}\right)\right|} \leq e^{a\left\|\left(V_{j}\right)_{j=1}^{\infty}\right\|_{\ell}}=e^{a\|V\|_{H^{2}\left(\mathbb{R}^{n}\right)}},
$$

the condition (21) yields $\mathbb{E} e^{a\left|\left(V_{1}, \ldots, V_{k}\right)\right|}<\infty$, implying that the moments, and hence the empirical correlations uniquely describe each measure $\mu^{J}\left(V_{1}, \ldots, V_{k}\right)$ with finite $J \subset \mathbb{N}$, as shown in [3].

Suppose that $W \in H^{2}\left(\mathbb{R}^{n}\right)$ is a random field that satisfies (21) and yields the same empirical correlations as $V$. In particular,

$$
\mu^{J}\left(V_{j_{1}}, \ldots, V_{j_{k}}\right)=\mu^{J}\left(W_{j_{1}}, \ldots, W_{j_{k}}\right),
$$

for every finite $J=\left\{j_{1}, \ldots, j_{k}\right\}$. It is a consequence of the Kolmogorov extension theorem $[50, \mathrm{Thm} .6 .16]$ that there is an unique probability distribution

$$
\mu^{\mathbb{N}}: \mathcal{B}\left(\mathbb{R}^{\mathbb{N}}\right) \rightarrow[0,1]
$$

in $\mathbb{R}^{\mathbb{N}}$, satisfying $\mu^{J}\left(V_{j_{1}}, \ldots, V_{j_{k}}\right)=\pi_{*}^{J} \mu^{\mathbb{N}}$ for any finite $J \subset \mathbb{N}$ where $\pi^{J}: \mathbb{R}^{\mathbb{N}} \rightarrow \mathbb{R}^{|J|}$ is the natural projection.

Finally, we can show that the probability distributions $V_{*} \mathbb{P}$ and $W_{*} \mathbb{P}$ equal as measures on Borel sets of $\ell^{2}$. Let $I: H^{2}\left(\mathbb{R}^{n}\right) \rightarrow \ell^{2}$ be the isometry $I:=\left(\left\langle\cdot, \varphi_{j}\right\rangle\right)_{j=1}^{\infty}$. It enough to prove the claim for elements $I(V)$ and $I(W)$. By uniqueness of $\mu^{\mathbb{N}}$,

$$
\iota_{*} I(V)_{*} \mathbb{P}=\mu^{\mathbb{N}}=\iota_{*} I(W)_{*} \mathbb{P},
$$

that is,

$$
I(V)_{*} \mathbb{P}\left(\iota^{-1} A\right)=I(W)_{*} \mathbb{P}\left(\iota^{-1} A\right)
$$

for every $A \in \mathcal{B}\left(\mathbb{R}^{\mathbb{N}}\right)$. Thus we obtain $I(V)_{*} \mathbb{P}=I(W)_{*} \mathbb{P}$ by Lemma 2.3. This completes the proof of Theorem 1.2.

\section{Appendix: Empirical Correlations on $\prod_{j=1}^{k} T \mathbb{S}^{n-1}$}

We shall show in detail how the representation of the empirical correlations $D^{k}(\mathbf{x}, \boldsymbol{\theta})$ on the tangent bundle $T \prod_{j=1}^{k} \mathbb{S}^{n-1}$ is constructed. Below, $\{\theta\}^{\perp}:=\left\{x \in \mathbb{R}^{n}: x \cdot \theta=0\right\}$ for $\theta \in \mathbb{S}^{n-1}$.

Lemma 3.1. The vector bundles $T \mathbb{S}^{n-1}$ and $\bigcup_{\theta \in \mathbb{S}^{n-1}}\{\theta\}^{\perp} \times\{\theta\} \rightarrow \mathbb{S}^{n-1}$ with the trivial projection $\operatorname{pr}_{2}(v, \theta):=\theta$ are isomorphic. The isomorphism is obtained from the tangent of the inclusion $\mathbb{S}^{n-1} \hookrightarrow \mathbb{R}^{n}$ by restricting the codomain.

Proof. By expressing each tangent vector $v \in T_{\theta} \mathbb{S}^{n-1}$ as an equivalence class of curves $\gamma$ : $(-\epsilon, \epsilon) \rightarrow \mathbb{S}^{n-1}$ with $\gamma(0)=\theta, \dot{\gamma}(0)=v$, the tangent map $T \iota: T \mathbb{S}^{n-1} \hookrightarrow T \mathbb{R}^{n}=\mathbb{R}^{n} \times \mathbb{R}^{n}$ associated to the inclusion $\iota: \mathbb{S}^{n-1} \hookrightarrow \mathbb{R}^{n}$ takes the form $T \iota[\gamma]=[\iota \circ \gamma]$. As $(\iota \circ \gamma)^{\prime}(0)$ is 
perpendicular to the base point $\iota \circ \gamma(0)$, we obtain $T \iota\left(T_{\theta} \mathbb{S}^{n-1}\right) \subset\{\theta\}^{\perp} \times\{\theta\}$ which, together with injectivity and the rank-nullity theorem, implies that $T \iota$ defines a bundle isomorphism

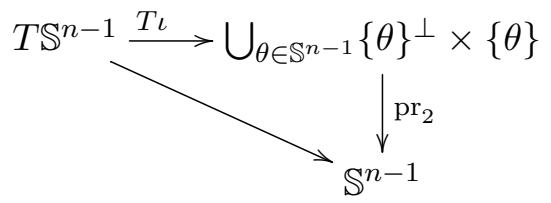

Note the untypical order of the base point $\theta$ and the fiber space $\{\theta\}^{\perp}$ in $\{\theta\}^{\perp} \times\{\theta\}$.

The empirical correlations can be rewritten as a generalised function $\boldsymbol{\theta} \mapsto D^{k}(\boldsymbol{\theta}) \in$ $\mathcal{D}^{\prime}\left(T_{\boldsymbol{\theta}} \prod_{j=1}^{k} \mathbb{S}^{n-1}\right)$ of the bundle $T \prod_{j=1}^{k} \mathbb{S}^{n-1}$ by setting

$$
\left\langle D^{k}(\boldsymbol{\theta}), \varphi\right\rangle=\left\langle D^{k},\left.\varphi \circ(T \iota)^{-1}\right|_{\prod_{j=1}^{k}\left\{\theta_{j}\right\}^{\perp} \times\left\{\theta_{j}\right\}}\right\rangle, \varphi \in C_{c}^{\infty}\left(T_{\boldsymbol{\theta}} \prod_{j=1}^{k} \mathbb{S}^{n-1}\right)
$$

where - with abuse of notation — we denote by $T \iota$ also the associated isomorphism

$$
\prod_{j=1}^{k} T \mathbb{S}^{n-1} \rightarrow \prod_{j=1}^{k} \bigcup_{\theta \in \mathbb{S}^{n-1}}\{\theta\}^{\perp} \times\{\theta\}
$$

generated by the tangent map above. It is a consequence of the invariance (3) that no information is lost in the identification. That is, the lift of $D^{k}(\cdot, \boldsymbol{\theta})$ to the space $T_{\boldsymbol{\theta}} \prod_{j=1}^{k} \mathbb{S}^{n-1}=$ $\prod_{j=1}^{k}\left\{\theta_{j}\right\}^{\perp} \times\left\{\theta_{j}\right\}$ is well defined and unique for each $\boldsymbol{\theta} \in \prod_{j=1}^{k} \mathbb{S}^{n-1}$.

\section{ACKNOWLEDGMENTS}

The authors would like to thank the referees for their valuable comments. PC is supported by BCAM-BERC 2018-2021 and SEV-2013-0323 and MTM2015-69992-R and Ikerbasque. TH is supported by Academy of Finland, project 275177. ML is supported by Academy of Finland, projects 312119 ( $\mathrm{CoE})$ and 303754. AK acknowledges support by Atmospheric Mathematics (ATMATH) project of University of Helsinki. and

\section{REFERENCES}

[1] Josselin Garnier and George Papanicolaou. Passive imaging with ambient noise. Cambridge University Press, 2016.

[2] Rakesh and Gunther Uhlmann. Uniqueness for the inverse backscattering problem for angularly controlled potentials. Inverse Problems, 30(6):065005, 24, 2014.

[3] Anatolij Dvurečenskij, Pekka Lahti, and Kari Ylinen. The uniqueness question in the multidimensional moment problem with applications to phase space observables. Reports on Mathematical Physics, 50(1):55 $-68,2002$.

[4] Liliana Borcea, George Papanicolaou, and Chrysoula Tsogka. Theory and applications of time reversal and interferometric imaging. Inverse Problems, 19(6):S139, 2003.

[5] Guillaume Bal, Tomasz Komorowski, and Lenya Ryzhik. Kinetic limits for waves in a random medium. Kinetic and Related Models, 3(4):529-644, 2010.

[6] Akira Ishimaru. Wave propagation and scattering in random media, volume 2. Academic press New York, 1978.

[7] Jean-Pierre Fouque, Josselin Garnier, George Papanicolaou, and Knut Solna. Wave propagation and time reversal in randomly layered media, volume 56. Springer Science \& Business Media, 2007.

[8] Maarten V De Hoop and Knut Solna. Estimating a green's function from "field-field" correlations in a random medium. SIAM Journal on Applied Mathematics, 69(4):909-932, 2009. 
[9] AJ Devaney. The inverse problem for random sources. Journal of Mathematical Physics, 20(8):1687-1691, 1979.

[10] Peijun Li and Ganghua Yuan. Stability on the inverse random source scattering problem for the onedimensional helmholtz equation. Journal of Mathematical Analysis and Applications, 450(2):872-887, 2017.

[11] Gang Bao, Chuchu Chen, and Peijun Li. Inverse random source scattering for elastic waves. SIAM Journal on Numerical Analysis, 55(6):2616-2643, 2017.

[12] Ming Li, Chuchu Chen, and Peijun Li. Inverse random source scattering for the helmholtz equation in inhomogeneous media. Inverse Problems, 34(1):015003, 2017.

[13] Gang Bao, Chuchu Chen, and Peijun Li. Inverse random source scattering problems in several dimensions. SIAM/ASA J. Uncertain. Quantif., 4(1):1263-1287, 2016.

[14] Gang Bao, Shui-Nee Chow, Peijun Li, and Haomin Zhou. An inverse random source problem for the Helmholtz equation. Math. Comp., 83(285):215-233, 2014.

[15] Peijun Li. An inverse random source scattering problem in inhomogeneous media. Inverse Problems, 27(3):035004, 22, 2011.

[16] Tapio Helin, Stefan Kindermann, Jonatan Lehtonen, and Ronny Ramlau. Atmospheric turbulence profiling with unknown power spectral density. Inverse Problems, 2018.

[17] Josselin Garnier and George Papanicolaou. Passive sensor imaging using cross correlations of noisy signals in a scattering medium. SIAM Journal on Imaging Sciences, 2(2):396-437, 2009.

[18] Josselin Garnier and Knut Sø lna. Background velocity estimation with cross correlations of incoherent waves in the parabolic scaling. Inverse Problems, 25(4):045005, 34, 2009.

[19] Josselin Garnier and Knut Sø lna. Transmission and reflection of electromagnetic waves in randomly layered media. Commun. Math. Sci., 13(3):707-728, 2015.

[20] Tapio Helin, Matti Lassas, Lauri Oksanen, and Teemu Saksala. Correlation based passive imaging with a white noise source. arXiv preprint arXiv:1609.08022, 2016.

[21] Liliana Borcea and Ilker Kocyigit. Imaging in random media with convex optimization. SIAM J. Imaging Sci., 10(1):147-190, 2017.

[22] Liliana Borcea, George Papanicolaou, and Chrysoula Tsogka. Adaptive interferometric imaging in clutter and optimal illumination. Inverse Problems, 22(4):1405-1436, 2006.

[23] Liliana Borcea, George Papanicolaou, Chrysoula Tsogka, and James Berryman. Imaging and time reversal in random media. Inverse Problems, 18(5):1247-1279, 2002.

[24] Otmar Scherzer, editor. Handbook of mathematical methods in imaging. Vol. 1, 2, 3. Springer, New York, second edition, 2015.

[25] Boris M. Shevtsov. Backscattering and inverse problem in random media. J. Math. Phys., 40(9):4359-4373, 1999.

[26] Matti Lassas, Lassi Päivärinta, and Eero Saksman. Inverse scattering problem for a two dimensional random potential. Comm. Math. Phys., 279(3):669-703, 2008.

[27] Tapio Helin, Matti Lassas, and Lassi Päivärinta. Inverse acoustic scattering problem in half-space with anisotropic random impedance. arxiv:1407.2481, 2015.

[28] Pedro Caro, Tapio Helin, and Matti Lassas. Inverse scattering for a random potential. arXiv preprint arXiv:1605.08710, 2016.

[29] David Colton and Andreas Kirsch. A simple method for solving inverse scattering problems in the resonance region. Inverse Probl., 12(4):383-393, 1996.

[30] Adrian I. Nachman. Reconstructions from boundary measurements. Ann. Math. (2), 128(3):531-576, 1988.

[31] R.G. Novikov. Multidimensional inverse spectral problem for the equation $-\Delta \psi-(v(x)-E u(x)) \psi=0$. Funct. Anal. Appl., 22(4):263-272, 1988.

[32] A.G. Ramm. Recovery of the potential from fixed energy scattering data. Inverse Probl., 4(3):877-886, 1988.

[33] G. Eskin and J. Ralston. The inverse backscattering problem in three dimensions. Comm. Math. Phys., 124(2):169-215, 1989.

[34] G. Eskin and J. Ralston. Inverse backscattering in two dimensions. Comm. Math. Phys., 138(3):451-486, 1991.

[35] Rakesh. Inverse problems for the wave equation with a single coincident source-receiver pair. Inverse Problems, 24(1):015012, 16, 2008. 
[36] Guanghui Hu, Jingzhi Li, and Hongyu Liu. Uniqueness in determining refractive indices by formally determined far-field data. Appl. Anal., 94(6):1259-1269, 2015.

[37] Hongyu Liu, Hai Zhang, and Jun Zou. Recovery of polyhedral scatterers by a single electromagnetic far-field measurement. J. Math. Phys., 50(12):123506, 10, 2009.

[38] Rakesh and William W. Symes. Uniqueness for an inverse problem for the wave equation. Comm. Partial Differential Equations, 13(1):87-96, 1988.

[39] Y. V. Kurylev and K. S. Peat. Hausdorff moments in two-dimensional inverse acoustic problems. Inverse Problems, 13(5):1363-1377, 1997.

[40] Y. V. Kurylev, N. Mandache, and K. S. Peat. Hausdorff moments in an inverse problem for the heat equation: numerical experiment. Inverse Problems, 19(2):253-264, 2003.

[41] Yaroslav Kurylev and Alexander Starkov. Directional moments in the acoustic inverse problem. In Inverse problems in wave propagation (Minneapolis, MN, 1995), volume 90 of IMA Vol. Math. Appl., pages 295323. Springer, New York, 1997.

[42] K. Schmüdgen. The Moment Problem. Graduate Texts in Mathematics. Springer International Publishing, 2017.

[43] J.J. Duistermaat. Fourier Integral Operators. Modern Birkhäuser Classics. Birkhäuser Boston, 2010.

[44] V. Petkov. Scattering Theory for Hyperbolic Operators. Studies in Mathematics and its Applications. Elsevier Science, 1989.

[45] Takahiro Shiota. An inverse problem for the wave equation with first order perturbation. American Journal of Mathematics, 107(1):241-251, 1985.

[46] A.G. Ramm and A.I. Katsevich. The Radon Transform and Local Tomography. Taylor \& Francis, 1996.

[47] S. Helgason. The Radon Transform. Progress in Mathematics. Birkhäuser Boston, 1999.

[48] J. Lohwater and O.A. Ladyzhenskaya. The Boundary Value Problems of Mathematical Physics. Applied Mathematical Sciences. Springer New York, 2013.

[49] F.G. Friedlander and M.S. Joshi. Introduction to the Theory of Distributions. Cambridge University Press, 1998.

[50] Olav Kallenberg. Foundations of modern probability. Springer Science \& Business Media, 2006. 